\title{
Artrogriposis neonatal asociada al uso de misoprostol
}

\author{
Neonatal arthrogryposis associated to misoprostol
}

\author{
Luis Enrique Torres-Batista, ${ }^{*}$ Fidel Enrique Torres-Batista, ${ }^{\ddagger}$ Idelvis Torres-Castañeda, ${ }^{\S}$ \\ Nancy de la Caridad-González, ${ }^{\S}$ Margarita Escalona-Fonseca ${ }^{\S}$ \\ * Hospital Provincial Ginecobstétrico "Fe del Valle Ramos”, Ministerio de Salud Pública, Manzanillo, Cuba; \\ ‡ Hospital Provincial Clínico-Quirúrgico Docente “Carlos Manuel de Céspedes”, Ministerio de Salud Pública, \\ Bayamo, Cuba; ${ }^{\S}$ Policlínico No. 2 “Ángel Ortiz Vásquez”, Ministerio de Salud Pública, Manzanillo, Cuba.
}

\begin{abstract}
RESUMEN
Introducción: La artrogriposis múltiple congénita (AMC) puede definirse como una displasia articular sistémica, caracterizada por rigidez articular en múltiples localizaciones de forma congénita. La incidencia es variable, puede diagnosticarse por ultrasonografía en edades tempranas de la gestación. Las posibles causas son numerosas. Presentación de caso clínico: Recién nacido con diagnóstico postnatal de AMC, ya que se identificaron deformidades articulares, limitación de movimientos, contractura de miembros inferiores, pie valgo convexo, astrágalo vertical bilateral, asociado con hernia inguinal congénita e hidrocefalia congénita. El paciente requirió evaluación de varias especialidades para su tratamiento. Hubo el antecedente de uso de misoprostol en los primeros meses del embarazo, lo cual podría ser la causa de la AMC. Conclusiones: Los pacientes con AMC pueden tener una gama de malformaciones, por lo que su reconocimiento temprano ayudará a ofrecer las mejores alternativas para mejorar, en lo posible, su pronóstico. Es indispensable la participación de especialistas en genética.
\end{abstract}

Palabras clave: Artrogriposis, recién nacido, misoprostol, malformaciones congénitas, hidrocefalia congénita.

\section{ABSTRACT}

Introduction: Congenital arthrogryposis multiplex (CAM) is a type of systemic joint dysplasia, characterized by congenital joint stiffness in multiple locations. Its incidence is variable, and it can be diagnosed by ultrasonography at early gestational ages. Multiple causes have been described. Case presentation: Newborn with postnatal diagnosis of CAM, since joint deformities, movement limitation, lower limb contractures, convex valgus feet, bilateral vertical talus, associated with congenital inguinal hernia and congenital hydrocephalus were identified. The patient required evaluation of several specialties for his treatment. There was a history of misoprostol use in the first months of pregnancy, which could be the cause of CAM. Conclusions: Patients with CAM can have a range of malformations, so their early recognition will help offer the best alternatives to improve their prognosis as much as possible. Participation of specialists in genetics is essential.

Keywords: Arthrogryposis, newborn, misoprostol, congenital malformations, congenital hydrocephalus.

Correspondencia: Luis Enrique Torres-Batista, E-mail: luise@infomed.sld.cu

Citar como: Torres-Batista LE, Torres-Batista FE, Torres-Castañeda I, De la Caridad-González N, Escalona-Fonseca M. Artrogriposis neonatal asociada al uso de misoprostol. Rev Mex Pediatr. 2021; 88(5): 199-203. https://dx.doi.org/10.35366/103901 


\section{INTRODUCCIÓN}

El desarrollo articular empieza secundariamente dentro de las condensaciones mesenquimales precoces del hueso cartilaginoso, aproximadamente alrededor de la quinta semana de gestación. Entre la séptima y octava semana aparecen los espacios articulares, así como movimientos de las extremidades, lo cual es esencial para el desarrollo normal. ${ }^{1}$

La artrogriposis múltiple congénita (AMC) es una enfermedad que se caracteriza por múltiples contracturas articulares, así como debilidad muscular. Habitualmente el diagnóstico es al nacimiento.

El término artrogriposis se deriva de las palabras griegas "arthro" (articulación) y "gryposis" (torcida). Este término incluye un conjunto de enfermedades en la que los niños afectados presentan al menos dos articulaciones rígidas en un mismo miembro, además de la rigidez de músculos de miembros superiores, inferiores y del dorso. Los músculos pueden estar ausentes, reducidos en tamaño o en número, o bien, estar reemplazados por tejido fibroso o grasa.

El grado de afectación varía de un paciente a otro; la deformidad clásica es bilateral y simétrica. Otto utilizó el término miodistrofia congénita en 1841. Stern usó la denominación de artrogriposis múltiple congénita en 1923. Sheldon usó por primera vez la palabra amioplasia congénita en 1992 relacionándola con una supuesta hipoplasia o aplasia muscular. Hall y colaboradores denominaron en 1983 a la amioplasia como la forma de AMC que afecta las cuatro extremidades. ${ }^{2,3}$

La incidencia de estos desórdenes se estima entre uno por cada 3,000 a 5,000 nacidos vivos. ${ }^{4}$ La mayoría de los niños afectados presentan al menos una lesión ortopédica que requiere atención médica. ${ }^{5}$ La forma de artrogriposis más frecuente es la amioplasia, con una incidencia de 1/10,000 nacidos vivos. ${ }^{6}$

En general, cualquier causa que lleve a la reducción de los movimientos fetales puede guiar a contracturas congénitas y, en casos graves, progresar a deformación. En los últimos 30 años se han identificado diferentes tipos de artrogriposis, así como las posibles causas, incluyendo las genéticas. ${ }^{7}$ Se han señalado también alteraciones en el desarrollo o funcionamiento de los músculos, del sistema nervioso central (SNC) o nervios periféricos, así como anormalidades neuromusculares de la placa terminal. Asimismo, se ha relacionado a limitación de espacio o restricción de movimiento en útero por oligoamnios, gestaciones múltiples, miomas o malformaciones uterinas. Además, por la exposición a misoprostol, penicilamina, inhibidores de la enzima convertidora de angiotensina (ECA), relajantes musculares. Por último, a enfermedades maternas: distrofia miotónica, miastenia gravis, diabetes mellitus, esclerosis múltiple. ${ }^{8,9}$

El misoprostol también se ha relacionado con el síndrome Moebius (SM), sugiriendo que tiene un efecto vasoconstrictor, que resulta en isquemia transitoria fetal afectando principalmente los núcleos de los nervios craneales abducens y facial. Asimismo, se ha propuesto que, al producir contracciones uterinas intensas y prolongadas, generaría reducción del flujo sanguíneo en ciertas áreas (por ejemplo: nervios craneales) y la restricción del crecimiento y movimiento fetal adecuado. ${ }^{10}$

En cuanto a los aspectos clínicos, en los pacientes con amioplasia, el tejido muscular es atrófico, el cual es reemplazado por tejido graso y fibroso. Las cuatro extremidades están involucradas en un patrón simétrico, y los individuos suelen tener inteligencia normal y no presentan malformaciones. ${ }^{11}$ Típicamente los pacientes se describen con hombros rotados internamente y aducidos, flexión palmar y contracturas de flexión de articulaciones interfalángicas distales, luxación de cadera, contracturas de extensión de rodillas y deformidad en pie equinovaro grave. ${ }^{12}$

El segundo tipo más común es la artrogriposis distal, una alteración con herencia autosómica dominante, que es un grupo de síndromes principalmente con contracturas articulares distales de las extremidades. Se han descrito al menos 10 tipos diferentes de artrogriposis distal. ${ }^{13,14}$

Los niños con AMC pueden presentarse con malformaciones congénitas mayores en corazón, pulmones, $\mathrm{SNC}$, aparato digestivo, entre otros. El pronóstico puede ser grave; por ejemplo, cuando se asocia con malformaciones múltiples puede producir muerte intraútero o en el periodo neonatal, por lo general secundario a hipoplasia pulmonar. ${ }^{15}$ En la forma clásica, el pronóstico está determinado por la limitación funcional que producen las contracturas articulares. ${ }^{16,17}$

El objetivo de esta comunicación es presentar el caso de un recién nacido $(\mathrm{RN})$ con $\mathrm{AMC}$ en quien además se identificó hidrocefalia congénita, pero que tenía antecedente de uso de misoprostol en las primeras etapas de la gestación, como posible factor causal.

\section{PRESENTACIÓN DEL CASO}

Recién nacido masculino, hijo de madre de origen Togole-Beninua de 20 años que vivía en la localidad de 
Kango de la ciudad de Libreville, capital de Gabón, en África. Antecedentes obstétricos: cuatro gestaciones, dos partos, un aborto espontáneo y tiene dos niños vivos; la madre no deseaba el embarazo y trató de abortar con el uso de misoprostol, pero sin lograrlo. Posteriormente, aceptó el embarazo. Recibió atención prenatal por enfermera obstetra (Sage-femme); asistió a algunas consultas y se le administró la vacuna antitetánica.

El parto ocurrió en junio de 2014 en el hospital de Kango, fue eutócico, de presentación cefálica, líquido amniótico claro. La evaluación del Apgar fue de $4 / 7$, con edad gestacional de 37.5 semanas y peso de 1,900 gramos. El personal de asistencia médica decidió trasladarlo al séptimo día de vida al servicio de Neonatología del Hospital Central Universitario de Libreville porque sospechaban que tenía múltiples malformaciones congénitas, y para ofrecer mejores cuidados médicos.

Por las condiciones generales, que se pueden observar en la Figura 1, se solicitan diversos estudios. En ecografía transfontanelar se identificó hidrocefalia congénita; mientras que la ecografía abdominal fue normal. Los especialistas de Ortopedia indican sólo fisioterapia. Por su parte, Neurocirugía sugiere intervención quirúrgica para colocar derivación ventrículo-peritoneal por la hidrocefalia congénita activa.

$\mathrm{Al}$ mes de nacido se realizó derivación ventrículoperitoneal sin complicaciones. Debido a que continuó con evolución favorable, se egresó para continuar se-

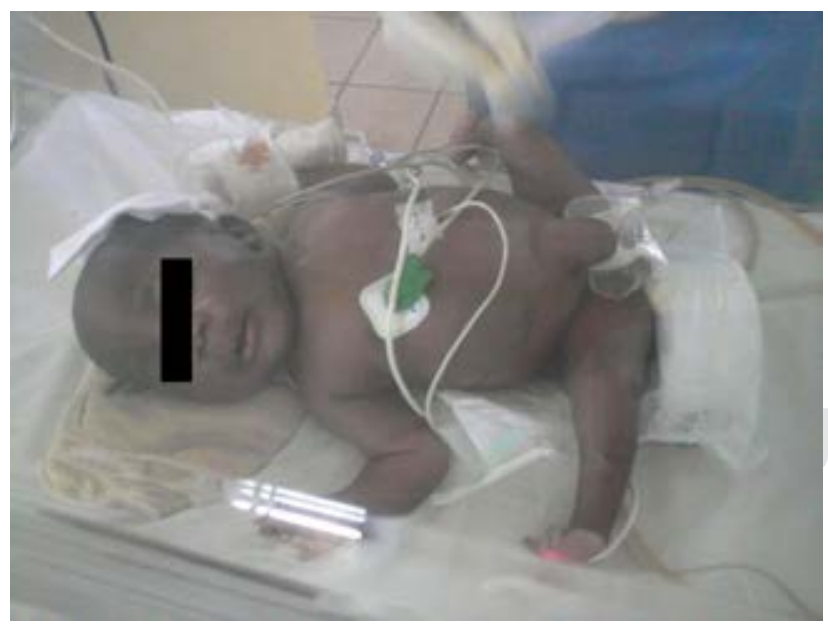

Figura 1: Fotografía tomada en las primeras horas de vida del recién nacido con artrogriposis múltiple, dentro de la incubadora. guimiento de manera ambulatoria por Neurocirugía, Neonatología y Ortopedia. Cabe señalar que no se realizaron estudios genéticos.

\section{DISCUSIÓN}

Según Hall, el término AMC fue acuñado para describir a RN con múltiples contracturas congénitas. ${ }^{18}$ La artrogriposis describe múltiples contracturas congénitas que son parte de más de 300 enfermedades diferentes. ${ }^{19,20}$ La etiología subyacente sigue siendo motivo de investigación. ${ }^{17,20}$

Se conoce que muchas mujeres, especialmente en países con leyes restrictivas para el aborto y con situación económica difícil, intentan terminar un embarazo temprano con misoprostol, aunque debido a la falta de información, lo utilizan en una variedad de formas, tanto de dosis como en etapas del embarazo. El misoprostol es una droga sintética análoga de la prostaglandina I 2, se utiliza en la práctica obstétrica para maduración cervical, aborto médico, evacuación del útero en caso de muerte fetal o embrionaria y en inducción del trabajo de parto. La exposición intraútero puede causar anomalías en el feto, siendo las más frecuentes los defectos en las extremidades con acortamiento o amputación de uno o más dedos, pies en equinovaro y alteraciones de los pares craneales. ${ }^{21}$ Misoprostol está reconocido como teratógeno, ya que causa constricciones anulares en miembros inferiores, AMC, hidrocefalia, holoprosencefalia, extrofia vesical y síndrome de Moebius. ${ }^{22}$

En el diagnóstico de contracturas múltiples congénitas o artrogriposis múltiple congénita, deben considerarse muchos más padecimientos que se clasifican según su presentación en:

1. Daño primario de extremidades ${ }^{23}$ como en el síndrome de Bruck, con incidencia menor a uno por cada millón de RN. Este padecimiento combina osteogénesis imperfecta y AMC, que se manifiesta con fracturas múltiples y recurrentes, así como con contracturas articulares congénitas con o sin pterigium, escoliosis y osteoporosis. Puede haber escleras azules y, por lo general, tienen dentinogénesis imperfecta o hipoacusia. ${ }^{24}$

2. Daño musculoesquelético más otra anomalía sistémica, ${ }^{10}$ como en el síndrome de Larsen, que es una alteración de la formación del tejido conectivo con patrón autosómico dominante y autosómico recesivo, que ocurre en 1:100,000 nacidos. Se caracteriza por la asociación de múltiples dislocaciones articula- 
res (cadera, rodillas y codos), y dismorfias faciales: abombamiento frontal, puente nasal deprimido, hipertelorismo y facies plana; además presentan deformidades en manos y alteraciones espinales. ${ }^{25}$ También puede haber síndrome de pterigium múltiple tipo Escobar, que se caracteriza por contracturas articulares en grados variables principalmente en cuello y grandes articulaciones. En cuanto a características faciales: facies sin expresión, paladar alto, boca pequeña, retrognatia y paladar hendido. ${ }^{26}$

3. Daño musculoesquelético más alteraciones del SNC o discapacidad intelectual o letal, ${ }^{23}$ como en el síndrome de Marden-Walker. Esta es una alteración en el tejido conectivo que incluye dismorfias faciales: facies tipo máscara, blefarofimosis, boca pequeña, micrognatia, paladar alto o hendido, pabellones auriculares de baja implantación. Además, los pacientes tienen múltiples contracturas articulares congénitas, masa muscular disminuida, talla baja, retraso psicomotor severo, así como anomalías renales, cardiovasculares o cerebrales. ${ }^{27}$

Por todo lo anterior, en los casos con AMC se recomienda la búsqueda etiológica específica, con la finalidad de establecer un consejo genético. Además, se requiere la participación de diversas especialidades para lograr mejorar el pronóstico, tanto de vida como funcional. ${ }^{6,28}$ En este último punto se debe destacar la importancia de iniciar la intervención ortopédica y la rehabilitación lo más temprano posible. La corrección apropiada de las contracturas requiere tratamiento ortopédico en la mayoría de los casos, lo que es complejo y con alta tasa de recurrencia. En general, estos pacientes requerirán de múltiples cirugías para liberar las contracturas y corregir las deformidades. Los procedimientos ortopédicos incluyen: yesos correctivos en las primeras etapas de la vida, liberaciones o alargamientos tendinosos, corrección del pie equinovaro o talo valgo, entre otros.

\section{REFERENCIAS}

1. Jones KL. Patrones reconocibles de malformaciones humanas. España: Elsevier Inc.; 2006. pp. 774-777.

2. Botha SJ, Butow KW. Gordon syndrome: literature review and a report of two cases. Cleft Palate Craniofac J. 2015; 52(1): e18-22.

3. Dieterich K, Le Tanno P, Kimber E, Jouk PS, Hall J, Giampietro $P$. The diagnostic workup in a patient with AMC: Overview of the clinical evaluation and paraclinical analyses with review of the literature. Am J Med Genet C Semin Med Genet. 2019; 181(3): 337-344. Available in: https://doi.org/10.1002/ajmg.c.31730

4. Obeidat MM, Audat Z, Khriesat W. Short-term functional outcome in children with arthrogryposis multiplex congenita after multiple surgeries at an early age. J Multidiscip Health. 2012; 5: 195-200.
5. Darin N, Kimber E, Kroksmark AK, Tulinius M. Multiple congenital contractures: birth prevalence, etiology, and outcome. J Pediatr. 2002; 140: 61-67.

6. Sucuoglu H, Ornek NI, Caglar C. Arthrogryposis multiplex congenita: multiple congenital joint contractures. Case Rep Med. 2015; 2015: 379730.

7. Hall J, Kiefer J. Arthrogryposis as a syndrome: gene ontology analysis. Mol Syndromol. 2016; 7(3): 101-109.

8. Nowlan NC. Biomechanics of fetal movement. Eur Cell Mater. 2015; 29: 1-21.

9. Valdés-Flores M, Casas-Avila Hernández-Zamora E, Kofman S, Hidalgo-Bravo A. Characterization of a group unrelated patients with arthrogryposis multiplex congenital. J Pediatr (Rio J). 2016; 92(1): 58-64.

10. Garamendi GP, Landa TM. Misoprostol as an abortive in Spain: report of a judicial autopsy case. Cuad Med Forense. 2004; 38 : 27-36.

11. Ma L, Yu X. Arthrogryposis multiplex congenita: classification, diagnosis, perioperative care, and anesthesia. Front Med. 2017; 11(1): 48-52.

12. Cassidy SB, Allanson JE. Arthrogryposis; management of genetics syndromes. In: Management of genetic syndromes. 3rd ed. WileyBlackwell; 2010, 81-96.

13. Kimber E. Amyoplasia and distal arthrogryposis. J Child Orthop. 2015; 9: 427-432.

14. Dhaubhadel S, Chapagain RH, Baniya B, Joshi H, Paudel KP. Bruck syndrome: a rare disorder in new-born with fractures and contractures. J Nepal Paediatr Soc. 2017; 37(3): 276-279.

15. Rodríguez-Ramos N, Capdevila-Leonori R, Nualart-Hernández L. Apertura del primer espacio en pacientes con artrogriposis múltiple congénita y pulgar aducto con técnica de colgajo dorsorradial del índice. Acta Ortop Mex. 2014; 28(1): 23-27.

16. Hoyos SV, Castrillón PS, Ortiz OI, Henao BJ. Artrogriposis en un hospital de alta complejidad de Pereira, Colombia. Revista Médica de Risaralda. 2014; 20(2): 121-124.

17. Dubousset J, Guillaumat M. Long-term outcome for patients with arthrogryposis multiplex congenita. J Child Orthop. 2015; 9: 449-458.

18. Wallach E, Walther-Louvier U, Espil-Taris C, Rivier, F, Baudoua E, Cances C. Arthrogryposis in children: etiological assessments and preparation of a protocol for etiological investigations. Archives de Pédiatrie. 2018; 25: 322-326.

19. Kowalczyk B, Felus J. Arthrogryposis: an update on clinical aspects, etiology, and treatment strategies. Arch Med Sci. 2016; 12(1): 10-24. Available in: http://doi.org/cc7k

20. Bjarne MM. Arthrogryposis multiplex congenita: an update. J Child Orthop. 2015; 9: 425-426.

21. Sánchez O. Guerra síndrome de Moebius, fetopatía por misoprostol. Reporte de un caso. Invest Clinic. 2003; 43: 1-5.

22. Opaleye ES, Coelho HL, Schuller-Faccini L, de Almeida PC, dos Santos EC, Ribeiro AJ et al. Evaluation of the teratogenic risks in gestations exposed to misoprostol. Rev Bras Ginecol Obstet. 2010; 32(1): 19-35.

23. Hall JG. Arthrogryposis (multiple congenital contractures): diagnostic approach to etiology, classification, genetics, and general principles. Eur J Med Genet. 2014; 57(8): 464-472.

24. Dhaubhadel S, Chapagain RH, Baniya B, Joshi H, Paudel KP. Bruck syndrome: a rare disorder in new-born with fractures and contractures. J Nepal Paediatr Soc. 2017; 37(3): 276279.

25. Mohammed MI, Mujammel H, Zahoor HD, Khatun SS, Imnul IM, Rahman SA et al. Larsen syndrome. Bangabandhu Sheikh Mujib Medical University. 2016; 9(2): 123-125. 
26. Mundios S, Horn D. Multiple pterygium syndrome, escobar variant In: Limb malformations. Berlin: Springer, 2014; 267. Available in: https://doi.org/10.1007/978-3-540-95928-1_60

27. Garavelli L, Donadio A, Banchini G, Magnani C, Magnani C, Calzolari E et al. Marden-Walker syndrome: case report, nosologic discussion and aspects of counseling. Genet Couns. 2000; 11(2): 111-118.
28. Gleason C, Juul S. Avery. Enfermedades del recién nacido. 10a ed. 2019. p. 1452.

Conflicto de intereses: Los autores declaran que no tienen. 